Relations industrielles

Industrial Relations

\title{
Grèves et lock-out au Québec - 1977, Rapport annuel, Québec, Direction générale de la recherche, ministère du Travail et de la Main-d'oeuvre, juin 1978, 43 pp.
}

\section{Jacques Mercier}

Volume 33, numéro 3, 1978

URI : https://id.erudit.org/iderudit/028901ar

DOI : https://doi.org/10.7202/028901ar

Aller au sommaire du numéro

Éditeur(s)

Département des relations industrielles de l'Université Laval

ISSN

0034-379X (imprimé)

1703-8138 (numérique)

Découvrir la revue

Citer ce compte rendu

Mercier, J. (1978). Compte rendu de [Grèves et lock-out au Québec - 1977,

Rapport annuel, Québec, Direction générale de la recherche, ministère du

Travail et de la Main-d'oeuvre, juin 1978, 43 pp.] Relations industrielles /

Industrial Relations, 33(3), 571-572. https://doi.org/10.7202/028901ar

Tous droits réservés (C) Département des relations industrielles de l'Université Laval, 1978
Ce document est protégé par la loi sur le droit d'auteur. L'utilisation des services d’Érudit (y compris la reproduction) est assujettie à sa politique d'utilisation que vous pouvez consulter en ligne.

https://apropos.erudit.org/fr/usagers/politique-dutilisation/ 
Ces brèves considérations ne peuvent, bien sûr, rendre compte convenablement d'un ouvrage de la qualité et de la densité de celui qui vient de nous être offert par J. Rojot. Il s'agit là d'un livre exemplaire de relations industrielles comparées, dans le véritable sens qui devrait être le leur. Exemplaire, c'est-à-dire pouvant être pris comme un modèle (en l'occurrence sur la manière dont on construit et on se sert d'un 'modèle' en relations industrielles); mais aussi, plus généralement, édifiant pour la voie à suivre par maints candidats auteurs de thèses de doctorat et de diverses autres recherches. De relations industrielles comparées, non seulement au plan européen, mais avec de fréquentes références aux États-Unis, pays que l'auteur connaît bien pour y séjourner périodiquement et pour $\mathrm{y}$ avoir soutenu sa thèse de $\mathrm{PhD}$ qui fut à l'origine de ce travail.

En gouvernant parfaitement la progression de son propos, Rojot démontre un art de la maïeutique qui le classe d'emblée parmi les maîtres. L'itération y est toujours pédagogiquement justifiée, sans jamais se transformer en redondance.

Un excellent livre, dont la lecture s'impose à tous ceux qui, spécialistes ou aspirant à le devenir, œuvrent dans le vaste et interdisciplinaire domaine des relations industrielles.

Dimitri WEISS

Université de Paris I Panthéon-Sorbonne

Grèves et lock-out au Québec - 1977, Rapport annuel, Québec, Direction générale de la recherche, Ministère du Travail et de la Main-d'œuvre, juin 1978, 43 pp.

Ce rapport annuel sur les Grèves et lock-out au Québec, 1977, le premier d'une série que la Direction générale de la recherche du ministère du Travail et de la Main-d'œuvre du Québec (MTMO) a entrepris de publier, fait suite au document méthodologique Grèves et lock-out au Québec, 1966-1976: quelques précisions sur les modes de compilations (octobre 1977) lequel fait l'objet d'une recension dans $\boldsymbol{R e}$ lations industrielles (volume 33, no. 1). Rappelons que le premier document com- portait des différences non-négligeables au niveau des définitions opérationnelles (e.g. durée d'un conflit et nombre de conflits) ainsi qu'au niveau des sources de collectes d'information d'avec celles utilisées par Travail Canada aux fins de la publication Strikes and Lock-Out in Canada.

Le présent rapport comprend quelques notes techniques, suivies de tableaux à deux entrées et finalement de la liste des conflits du travail déclenchés et en vigueur au Québec en 1977. Le rapport évite en bonne partie le problème du chevauchement des statistiques en présentant un niveau de précision et de désagrégation qu'on ne retrouve pas dans le document fédéral. Les tableaux III (conflits vs secteurs d'activités et juridictions), IV (conflits vs régions administratives), et $\mathrm{V}$ (conflits selon l'affiliation syndicale) seront particulièrement appréciés par l'analyste de la scène québécoise des relations industrielles. Le tableau I innove en distinguant entre grèves et lock-out et entre conflits légaux et illégaux. On trouvera également quelques autres innovations dans l'organisation des données présentées (plus grand détail au niveau du secteur public, etc.). Le tableau II quant à lui présente huit indices globaux reliés aux conflits du travail pour 1976, première année de production par le MTMO, et 1977. Il aurait été intéressant pour le lecteur de retrouver également dans les autres tableaux les données de 1976 pour des fins de comparaisons.

Il apparaîtrait également essentiel que les principaux indices sur les conflits du travail en provenance de chaque source de diffusion (Travail Canada et MTMO) se retrouvent dans ce rapport. En effet, pour des raisons méthodologiques mais également parce qu'il a amélioré la couverture des conflits du travail au Québec, le MTMO offre des chiffres qui diffèrent substantiellement parfois des données fédérales. Par exemple, il est évident que l'inclusion de la manifestation du 14 octobre 1976 dans les données du MTMO explique pour une bonne part les écarts au niveau du «nombre de travailleurs touchés» et que la différence dans la définition de la durée d'un conflit explique pour sa part l'écart dans le «nombre de conflits» recensés. De telles différences dans les indices se retrouvent également parmi les secteurs d'activités. 
Le problème de la comparabilité des données ne se pose pas que dans le cas des études de comparaisons internationales ou interrégionales. C'est davantage dans l'utilisation quotidienne qui en sera faite par les divers interlocuteurs du monde des relations industrielles que la difficulté apparaît. Par ignorance ou par intérêt, il est facile d'exploiter de telles différences et de semer la confusion.

Mais c'est là un problème qui est sans doute appelé à disparaître graduellement dans la mesure où chaque agence de difsusion permettra à son homologue d'obtenir une plus grande rigueur. En ce sens, il faut se féliciter du coup de barre que le MTMO vient de donner dans tout le processus de production des données sur les conflits du travail. On apprend d'ailleurs dans l'avant-propos du rapport que l'envoi d'un questionnaire à tous les employeurs touchés par un conflit du travail est envisagé pour 1978 afin d'uniformiser et de détailler davantage (il s'agit en fait d'une étape additionnelle dans le processus de cueuillette des données) l'information. C'est là une démarche des plus intéressantes et il faut espérer qu'un effort sérieux de concertation et d'uniformisation se fera entre la MTMO et Travail Canada. afin d'en tirer tout le bénéfice possible.

Jacques MERCIER

Université Laval

Academic Bargaining: Origins and Growth, by James P. Begin, Theodore Settle and Paula Alexander, New Brunswick, New Jersey, Rutgers University, 1977, 232 pp.

Le volume se situe dans le cadre d'une vaste recherche sur les facteurs qui ont contribué à la syndicalisation professorale en milieu universitaire et ses effets sur la structure et le processus décisionnel de ses institutions. Le titre est un peu trompeur, puisqu'il s'agit ici d'une analyse détaillée de la situation dans l'État du New Jersey seulement. Par contre, il faut ajouter que cet État est un champ privilégié d'analyse. Le New Jersey fut le premier État américain où la négociation collective dans le système d'enseignement public fut établie à tous les niveaux. C'est égalemen dans cet état que la négociation collective fut reconnue pour la première fois dans un collège privé, soit le Monmouth College en 1971. C'est en vertu des lois fédérales du travail que l'accréditation syndicale fut accordée dans ce cas; ce qui renversait dix-huit années de précédents du Bureau National des Relations de Travail.

Les auteurs présentent leur ouvrage en deux parties. Ils commencent par nous situer dans l'État du New Jersey et dans son système scolaire. Dans un deuxième temps, ils étudient le cas spécifique de plusieurs institutions universitaires, dont l'Université Rutgers, les Collèges communautaires, le Monmouth College etc. La deuxième partie est donc une étude de neuf cas distincts. Les facteurs de satisfaction et de motivation sont étudiés selon trois grands schèmes de variables: 1) les variables externes au milieu universitaire qui influencent directement leurs opérations telles les lois ouvrières, les luttes syndicales; 2) les caractéristiques organisationnelles de chacune des universités et l'évolution qu'elles ont subies au cours des années ' 60 ; et 3 ) les caractéristiques spécifiques du corps professoral et de l'administration de chacune des universités.

La situation au New Jersey présente d'intéressantes similitudes avec celle du Québec. Entre autre, on peut signaler $1^{\circ}$ la création d'une Commission d'étude au début des années ' 60 qui commanda la mise sur pied d'un régime de collèges régionaux avec chacun, son bureau de direction; $2^{\circ}$ la création, en 1966, d'une Commission de l'enseignement supérieur à l'égard de laquelle certaines universités exprimèrent plusieurs réserves en tentant de protéger leur autonomie; $3^{\circ}$ l'intégration des anciennes écoles de formation des maitres dans le réseau universitaire et $4^{\circ}$ la mise en place d'une législation permettant la syndicalisation dans le secteur public.

L'analyse nous permet de dégager assez clairement que la syndicalisation des collèges régionaux a engendré un effet d'entraînement parmi les institutions publiques, même si parfois le mouvement est clairement défensif. De plus, les auteurs constatent qu'en soit la reconnaissance législative n'a pas accéléré le processus de syndicalisation. Par ailleurs, la méfiance à l'égard de la nouvelle Commission de l'En- 